EOMmun Communication et organisation

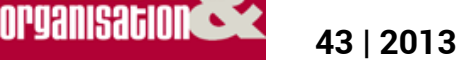

Réseaux sociaux entre médias et médiations

\title{
La représentation sociale du risque de précarité chez les étudiants
}

Sophie Béguinet

\section{OpenEdition}

1 Journals

Édition électronique

URL : http://journals.openedition.org/communicationorganisation/4236

DOI : 10.4000/communicationorganisation.4236

ISSN : 1775-3546

Éditeur

Presses universitaires de Bordeaux

Édition imprimée

Date de publication : 1 juin 2013

Pagination : 227-252

ISBN : 978-2-86781-883-7

ISSN : 1168-5549

Référence électronique

Sophie Béguinet, «La représentation sociale du risque de précarité chez les étudiants »,

Communication et organisation [En ligne], 43 | 2013, mis en ligne le 01 juin 2015, consulté le 10

décembre 2020. URL : http://journals.openedition.org/communicationorganisation/4236 ; DOI :

https://doi.org/10.4000/communicationorganisation.4236 


\title{
La représentation sociale du risque de précarité chez les étudiants
}

\author{
Sophie Béguinet ${ }^{1}$
}

La précarité est une notion polymorphe. Si la recherche ne s'accorde pas sur sa définition, il n'y a pas plus de consensus en politique. Pourtant, tout le monde s'accorde à dire que la précarité augmente, et les facteurs de risque identifiés se multiplient. De nouvelles formes de précarités sont ainsi mises à jour, en témoigne l'apparition d'une nouvelle forme de ce risque, la précarité énergétique. Certaines catégories de population sont identifiées comme étant plus sensibles au risque de précarité.

Nous nous intéresserons pour notre part aux étudiants de l'enseignement supérieur. Les étudiants sont des oubliés de la catégorisation politique et administrative, car ils n'ont pas encore intégré le marché du travail. La précarité restant fortement liée à sa dimension "emploi ", les étudiants ne sont donc pas considérés par les pouvoirs publics comme des populations fragiles, sensibles à ce risque. En témoigne Benoist Apparu, secrétaire d'État au logement : «J'entends beaucoup de discours sur : « la précarité étudiante se développe $» . .$. J'attends encore qu'on me le démontre $»^{2}$.

Pourtant, les associations, intermédiaires privilégiés des pouvoirs publics pour appréhender les besoins des usagers et citoyens, dénoncent la montée de la précarisation des étudiants. Ils relèvent que de plus en plus d'étudiants font appel à eux pour subvenir à leurs besoins, ce qui, pour Marc Castille, directeur des relations extérieures et institutionnelles du Secours populaire, est assez alarmant : " Pour nous, c'est vraiment la face émergée de l'iceberg [...] On imagine bien que pour un jeune étudiant, venir frapper à la porte du Secours populaire, des Restos du cœur ou de l'Armée du salut, ce n'est quand même pas une démarche d'avenir, il faut vraiment être au bout du rouleau $»^{3}$. Cette situation ne concerne pas uniquement les «grandes» villes

\footnotetext{
1 ATER en Sciences de Gestion, CREG, Université de Pau, sophie.beguinet@univ-pau.fr 2 Émission Une Semaine d'Enfer, France 4, 19 octobre 2011.

3 Source : Reuters http://fr.reuters.com/article/topNews/idFRPAE7BM06Z20111223?pageNumber=2\&virtu alBrandChannel=0 [consulté le 19 juin 2012].
} 
universitaires : à Pau, l'épicerie solidaire a dû ouvrir un deuxième jour en semaine pour répondre à la demande.

Le risque de précarité est important chez les étudiants, notamment au regard leur degré de dépendance/indépendance vis-à-vis de la sphère familiale. D’un côté l'étudiant indépendant, qui s'assume entièrement seul et doit concilier travail à temps partiel et études pour subvenir à ses besoins. De l'autre, l'étudiant dépendant, qui vit encore au domicile familial et se retrouve donc soumis aux aléas de la vie familiale. Entre ces deux extrêmes, une multitude de situations individuelles, qui résultent des interactions et combinaisons d'une large palette de critères ayant trait au logement, aux revenus, à l'alimentation, etc. Le risque de précarité résulte alors de l'agrégation de ces multiples facteurs de risque. L'articulation de ces critères fait qu'il y a autant de situations à risque que d'étudiants précaires.

Nous avons choisi de nous intéresser aux représentations sociales de la précarité chez les étudiants, pour révéler les facteurs de risque qui sous-tendent sa survenance dans la vie de ces individus. C'est le cumul de ces facteurs de risque et leurs agencements qui vont augmenter la probabilité d'entrer dans un processus de précarisation.

Lintérêt d'une telle étude est de mettre en évidence, pour une population reconnue fragile, les dimensions qu'elle identifie comme caractéristiques d'un risque. Les études sont un temps de construction de l'identité personnelle et sociale nous dit Erlich (1998). S'intéresser aux représentations du risque de précarité chez les étudiants, c'est alors mettre à jour la pensée sociale d'un groupe qui constituera demain la société française avec ses normes, valeurs et croyances, pour ainsi se donner les moyens de gérer leur risque de précarité. En effet, la révélation des facteurs de risque de précarité par la population étudiante soulignerait alors ce qu'elle considère comme déclencheur d'un processus de précarisation. Cette connaissance pourrait alors permettre d'agir en amont de la précarisation, pour protéger si ce n'est prévenir son amorçage chez les étudiants.

L'objet de cette étude est ainsi à la fois de clarifier la notion de précarité, pour mettre en évidence que sa multidimensionalité et son caractère processuel ont contribué à la naissance d'un risque social majeur, et de mettre en évidence les facteurs de risque principaux de la précarité, par l'analyse des représentations sociales d'un groupe social reconnu vulnérable à ce risque, les étudiants. Pour dévoiler ces représentations sociales, nous mobiliserons la théorie du noyau central de Moscovici (1976) et Abric (2011) pour opérer une analyse prototypique (Vergès, 1992) permettant de repérer le contenu et la structure de celles-ci. Puis nous approfondirons cette connaissance de la représentation du risque de précarité chez les étudiants en procédant à une analyse de contenu. Nous présenterons ensuite les résultats que nous discuterons, puis nous conclurons par les limites et principaux enseignements 
de cette étude afin de présenter des préconisations de protection des étudiants précaires.

\section{La précarité, une instabilité facteur de risque de pauvreté et d'exclusion}

Dans le sens actuel, la précarité est fréquemment réduite à la précarité de l'emploi. Or le sens de ce terme est bien plus large, et traduit à l'origine un risque de pauvreté et d'exclusion.

Etymologiquement, «précarité» vient du latin «precarius», désignant ce qui s'obtient par la prière et qui par conséquent ne s'obtient que par la grâce d'autrui. Cela renvoie à une certaine forme de dépendance, de domination dans les rapports sociaux et " être en situation de précarité renvoie alors à l'absence de choix et à la faiblesse des marges de manœuvre dans la conduite de l'existence » (Hélardot, 2005). Cette dépendance à autrui trouve sa source dans le manque, les carences qui ponctuent la vie d'un individu dans ses divers pans tels que : santé, emploi, revenus, éducation, logement, lien social, etc. Cette première approche sémantique, rappelle les notions de pauvreté et exclusion, lesquelles diffèrent de la notion de précarité mais continuent d'en imprégner sa représentation.

Les premières recherches sociologiques sur la précarité datent de la fin des années 1970 et s’inscrivent dans la sociologie de la famille et de la pauvreté. En effet, dans un contexte où le taux de chômage, autour de $5 \%$, paraît encore «maîtrisable» (Baverez, 1996 ; Malinvaud, 1986), la notion de précarité n'est pas encore principalement centrée sur l'emploi. La sociologie va alors s'intéresser à la forte corrélation entre pauvreté, exclusion et précarité, tout en prenant garde de distinguer ces trois notions. Les trois notions coexistent tout en se recoupant. À l'instar de la précarité, la pauvreté et l'exclusion sont des notions multidimensionnelles et contextuelles, dont les signes peuvent être appréciés selon divers critères.

La différence majeure entre d'une part la précarité, et d'autre part la pauvreté et l'exclusion est que ces dernières peuvent être définies par défaut, c'est-à-dire par rapport à un état «normal» qui va être considéré comme un point de basculement dans l'un ou l'autre de ces états. De nombreux seuils ont été déterminés pour la pauvreté, l'exclusion restant plus appréciable qualitativement, par rapport à ce qui est «inclus», «inséré», dans un contexte donné. Il en résulte que l'on est pauvre, que l'on est exclu selon les critères retenus, dans un environnement donné. Et il en va de même pour la précarité.

Toutefois, contrairement à ces deux notions, la précarité ne marque pas un état, c'est une situation provisoire, une transition vers une meilleure/pire condition. Les notions de pauvreté et d'exclusion intègrent une dimension statique qui marque l'individu jusque dans son identité : les pauvres/exclus constituent son groupe d'appartenance, et les croyances, symboles, valeurs qui traversent ce groupe vont progressivement devenir siens. À l'inverse, « à l'espace du précaire, mobile et discontinu, semble correspondre le paradoxe 
d'une identité construite sur du "provisoire" [...] Le précaire est seul, différent, éradiqué dans son rapport à l'espace »(Offredi, 1988, in Barbier, 2005). Dès lors, «les précaires» ne forment pas un groupe homogène, auquel on peut associer une identité collective. Le précaire c'est le chômeur, le parent isolé, le jeune qui termine ses études, le divorcé, etc. Il n'y a pas de figure du précaire, c'est un individu à l'identité incertaine (Ehrenberg, 1999). Il est alors extrêmement difficile de repérer l'amorçage d'un processus de précarisation. Cette lacune tient en partie à la définition même de la précarité, à sa confusion avec ces deux notions proches, entretenue par l'usage quotidien du terme.

Si la précarité emprunte des éléments de signification à la pauvreté et l'exclusion, elle ne les recouvre pas totalement. Ces trois notions restent toutefois intimement liées, la précarité traduisant un processus qui peut conduire à l'exclusion et à la pauvreté. Mais cette relation n'est pas nécessaire : la précarité est un facteur de risque de pauvreté et d'exclusion, la probabilité d'atteindre l'un de ces deux états augmentant au fil de la précarisation.

Par ailleurs, une situation précaire évoque spontanément une situation instable. Précarité et instabilité sont fréquemment utilisées dans le langage courant comme synonymes. Cette instabilité est porteuse d'une deuxième signification qui marque la notion de précarité : l'incertitude.

Alors que l'appréciation de l'instabilité s'effectue a posteriori, via un retour sur expérience permettant à l'individu de constater qu'à telle période il se trouvait dans une situation instable, dans l'instant, ce n'est pas tant l'instabilité que l'inconfort d'une situation qui marque l'individu. Cette gêne trouve sa source dans l'incertitude à laquelle fait face l'individu concernant son avenir plus encore sur le court terme, son lendemain. Avec cette dimension temporelle, traduisant à la fois un présent instable et un avenir incertain, cela confirme que la précarité se définit moins comme état de fait, une catégorie fixée selon des critères objectifs et donc statistiquement mesurable, que comme la résultante d'une multiplicité d'expériences touchant à la précarité. La précarité se définit comme un processus et renvoie à une insécurité multiforme (Boumaza et Pierru, 2007) qui affecte l'individu dans sa trajectoire de vie.

Repérer la précarité n'est alors pas chose aisée puisque son identification demande l'intégration de la dimension diachronique des situations étudiées qui sont, par définition, amenées à évoluer (Hélardot, 2005). La précarité concerne des populations hétérogènes, résidant dans divers territoires, et se décline dans de nombreux domaines, lesquels s'articulent presque de manière unique, en fonction des situations individuelles. Cette diversité montre toute la complexité à laquelle font face les pouvoirs publics dans leur volonté de venir en aide à ces personnes en difficulté. En effet, il existe moins une précarité que des précarités (Sanchou, 2005). De plus, il y a une refonte permanente de la définition de la précarité, laquelle révèle autant qu'elle produit les nouvelles formes de précarité et les signes associés à cette notion qui semble extensive à volonté. 
Nébuleuse et pourtant hautement symbolique des problèmes sociaux contemporains, la précarité résonne comme une notion fédératrice contre laquelle chaque individu touché se doit de se mobiliser : problèmes de logement, chômage, carences en terme de protection sociale, etc., tous peuvent se retrouver sous le label de la précarité et s'unir pour y faire face. La notion de précarité revêt ainsi un caractère subi. L'individu isolé, l'intérimaire, le parent isolé, ne sont pas précaires si leur mode de vie résulte d'un choix librement effectué. En cela, l'approche de la précarité en tant que péril est une définition bien française. Les Anglo-Saxons lui préfèrent ainsi le terme "flexibility", gardant ainsi cette idée d'instabilité mais lui donnant une connotation beaucoup plus positive mobilisant les champs lexicaux de la mobilité, de l'adaptabilité, du dynamisme, ou encore du changement. La "précarité à la française» est ainsi une notion inexportable car elle ne trouve aucun équivalent conceptuel à l'étranger; de fait, les approches comparatives du phénomène sont impossibles (Barbier, 2005).

Le glissement de la notion vers une signification liée à l'emploi n'apparaît que vers la fin des années 1980, lorsque les signes alarmants d'un taux de chômage qui ne cesse de croître et les conséquences dramatiques d'un chômage de masse $e^{4}$ structurel sont identifiés. Les travaux des sociologues sur la précarité liée à l'emploi vont se multiplier pour la relier étroitement au chômage, aux emplois à statuts particuliers, etc. Un facteur de risque est enfin identifié, et permet au discours politique de s'attaquer à ce problème (réglementations, dispositifs, droits sociaux, etc.). Relayé par l'administration dans les textes, relayé dans la population par les médias, "précarité» s'utilise pour "précarité de l'emploi», occultant ainsi la diversité des situations recouvertes par ce terme. L'ancrage de la précarité dans une signification liée à l'emploi est encore très présent aujourd'hui dans les représentations.

La construction sociale du risque de précarité s'amorce alors, et s'accompagne de la mise à jour de multiples facteurs de risque conduisant à définir la précarité comme un risque multidimensionnel. D'une situation de dépendance et d'instabilité, productrice d'incertitude, la précarité devient un risque qu'il faut gérer afin de prévenir l'amorçage de son processus ou protéger les individus déjà précaires.

\section{La précarité, naissance et ancrage d'un risque social majeur}

En France, la progression et l'installation durable de la précarité en tant que problématique sociale trouve sa source dans les bouleversements sociétaux amorcés dans les années 1970 : la désindustrialisation, la mondialisation, le

4 Le chômage structurel de masse émerge en fait dans les années 1930 (krach boursier), mais c'est dans les années 1970 (chocs pétroliers) qu'il prend des proportions considérables. Inférieur à $2 \%$ durant toute la période de l'après-guerre jusqu'en 1967 (Malinvaud, 1986), le taux de chômage commence son ascension : « lent à l'origine (2,8 \% en 1974 contre 1,6\% en 1966), le mouvement s'accéléra à partir de 1974, alternant trois périodes de hausse rapide en 1974-1975 (2,8 à 4 \%), 1981-1986 (7,4à 10,4 \%), puis 1990-1994 (8,9 à 12,5\%) » (Baverez, 1996). 
ralentissement de la croissance économique, le développement des nouvelles technologies de l'information, sont autant de facteurs qui ont profondément modifié le visage de la société. Les grandes institutions sur lesquelles s'appuyaient autrefois les individus ne jouent plus leur rôle intégrateur : l'État, l'Église, la Famille et le Travail, qui structuraient la vie des individus (identité, rôle, statut, etc.) et régulaient le fonctionnement de la société, s'effritent au profit d'un mode de fonctionnement individualiste autonome (au niveau micro) et d'une organisation basée sur la logique économique de marché (au niveau macro).

La société se pose comme le produit de la mondialisation de l'économie, avec ses exigences de flexibilité, de mobilité, d'adaptabilité aux changements permanents, induisant ainsi une modification profonde de nos comportements. Le rapport au temps s'est accéléré et il n'est plus question de vivre dans le moment, mais plutôt dans l'urgence. Être efficace ne suffit plus, il faut être efficient, il faut être performant (Ehrenberg, 2008).

La cause profonde de cette nouvelle relation de l'individu au temps provient des importants bouleversements économiques survenus ces dernières années. La société contemporaine ayant été gagnée par une logique de marché globalisé s'appuyant sur des moyens de communication bigh-tech, le temps s'est considérablement contracté, passant du long terme au court terme pour finir par une conception de l'instantané. La société avance en temps réel, et (en)traîne dans son sillon les individus qui ne fonctionnent plus que dans l'instant, l'immédiat, l'urgent. Contraint par ce rythme effréné, l'individu ne parvient plus à s'extraire de cette emprise et l'on voit alors émerger une société où tout semble possible mais qui rejette impitoyablement ceux qui ne parviennent pas à suivre ses exigences (Aubert, 2004).

Outre la multiplication de nombreuses pathologies telles que le stress, l'épuisement ou la dépression (Ehrenberg, 2000), les conséquences socio-économiques sont dramatiques : chômage de masse, accroissement des inégalités, renforcement mais aussi apparition de nouvelles problématiques sociales, comme par exemple celle des travailleurs pauvres (Beck, 1998; Clerc, 2009). Les nouvelles réalités socio-économiques sous-tendent tout autant qu'elles encouragent une recrudescence globale de diverses formes de précarités, notamment dans le monde du travail où les salariés sont alors de plus en plus isolés, impuissants face à ce rythme qu'ils subissent. La France connaît en effet à l'époque l'une des plus fortes croissances de son taux de chômage ${ }^{5}$, et Castel $(1999,2003)$ la relie à la déstabilisation générale de la société, notamment au travers du prisme de la précarité du travail analysée sous l'angle des mutations du rapport salarial. L'accent est mis sur l'effritement général de la condition salariale, cette précarisation massive du travail touchant l'ensemble des travailleurs, et ses conséquences en

5 En l'espace de 30 ans, le taux de chômage en France a progressé de 10 points : de 1,6 \% en 1966 à 12,5\% en 1994 (Baverez, 1996). 
terme de vulnérabilités socio-économiques, comme par exemple le chômage, l'exclusion, la désaffiliation.

Pour Beck (2008) et Giddens (2000), ces mutations sociétales traduisent le passage à une société de risques, où le risque devient un facteur intrinsèque, produit par la société et l'activité humaine (Beck, 2008 ; Giddens, 2000). L'instabilité s'érige comme un fondement, et tous les pans de la société en sont désormais affectés : la société se précarise et avec elle, les individus. La société du risque ne signifie pas que la société soit plus dangereuse aujourd'hui qu'hier, mais que leur présence dans notre imaginaire s'est accrue (Peretti-Watel, 2000). Notre rapport au danger s'est mué en un refus de nombreuses formes de risque, qui ont alors été mises à jour afin d'être gérées. De cette société en pleine mutation, va se former un nouveau risque social, la précarité, qui requiert une protection toujours plus efficace des pouvoirs publics. La précarité se transforme alors en une incertitude probabilisable, un risque qu'il faut gérer.

Le terme "précarité» apparaît alors de manière récurrente dans le discours politique, dès la fin des années 70. Partis politiques, organisations syndicales, etc., tous s'approprient cette thématique et cela entraîne une confusion relativement importante sur la définition de la précarité. Sans trop savoir ce que recouvre ce terme (chômage ? pauvreté ? exclusion ?), il en ressort une connotation négative stigmatisant ces précaires qu'il faut absolument sauver, un problème social majeur qu'il faut absolument combattre. Tout naturellement le droit s'empare alors de la notion pour transformer cette catégorie politique en une catégorie juridique utilisée par l'administration, permettant ainsi de définir, d'identifier et d'ouvrir des droits sociaux à cette population désormais reconnue fragile. L'article 2 de l'arrêté ministériel de 1992 reconnaît ainsi cinq catégories de personnes qu'il faut protéger de ce risque social : les chômeurs, les bénéficiaires du RMI, les titulaires d'un contrat emploi solidarité, les personnes sans domicile fixe, et les jeunes âgés de 16 à 25 ans exclus du milieu scolaire et engagés dans un processus d'insertion professionnelle. De 1975 à 1988 (année marquée par la loi sur le RMI), on peut déjà recenser 77 textes administratifs relatifs aux questions de pauvreté et précarité (Barbier, 2005). La précarité est alors surtout perçue dans sa dimension économique et éminemment liée à l'emploi.

En ne s'attachant pas à l'observation des seuls aspects économiques, la sociologie révèle les insuffisances de la catégorisation politique et de fait l'existence de ces nombreux précaires qui ne correspondent pas aux critères de définition utilisés pour l'ouverture de droits sociaux. Ces précaires «insoupçonnés» ont un emploi et un cadre familial stables, mais vont par exemple souffrir d'une carence ou d'une difficulté d'accès à la connaissance. Un rapport du Conseil économique et social vient dès 1987 éclairer la définition de la précarité pour enfin la considérer dans ses multiples dimensions, permettant ainsi au politique d'élargir sa vision du phénomène et d'en 
saisir toute la complexité : «la précarité est l'absence d'une ou plusieurs des sécurités permettant aux personnes et familles d'assumer leurs responsabilités élémentaires et de jouir de leurs droits fondamentaux. Linsécurité qui en résulte peut être plus ou moins étendue et avoir des conséquences plus ou moins graves et définitives. Elle conduit le plus souvent à la grande pauvreté quand elle affecte plusieurs domaines de l'existence, qu'elle tend à se prolonger dans le temps et devient persistante, qu'elle compromet gravement les chances de reconquérir ses droits et de réassumer ses responsabilités par soi-même dans un avenir prévisible. » (Wresinski, 1987).

La précarité s'érige alors comme un risque social majeur, révélateur de la société française contemporaine. Elle suscite une mobilisation massive et appelle l'individu autant que la société à s'interroger sur les défis économiques, technologiques, sociaux, et politiques à relever pour affronter l'avenir. Afin de définitivement s'émanciper de la vision restrictive de la précarité de l'emploi, un score de précarité individuelle a été mis au point pour permettre aux CES (Centres d'examens de santé) d'identifier plus finement les personnes à risque de précarité (Moulin et al., 2006; Sass et al., 2006). Fruit d'un lourd dispositif de recherche, le score Epices (Évaluation de la précarité et des inégalités de santé pour les CES) permet de s'intéresser aux populations en situation de précarité autrement que par le prisme économique/financier et ses indicateurs trop restrictifs (les catégories administratives définies par l'arrêté de 1992 ne sont en effet plus les seules populations concernées). Reposant sur seulement 11 questions binaires, le score Epices permet de calculer la probabilité d'entrer dans un processus de précarisation, le risque de précarité augmentant selon le score obtenu.

Incertitude désormais probabilisable, la précarité se définit alors comme un processus dynamique multidimensionnel, fruit de l'articulation d'une succession d'événements subis dans les différents pans de la vie d'un individu (emploi, travail, famille, logement, santé, éducation...). Ce risque se présente comme une insécurité multiforme, résultant du cumul de facteurs de risque qui s'agencent et interagissent de manière dynamique. La probabilité d'entrer dans un processus de précarisation est alors omniprésente car ses facteurs de risque sont présents au quotidien dans la vie de l'individu : la probabilité d'être renvoyé, de ne pas pouvoir payer ses factures ou encore de se séparer de son conjoint... sont autant de facteurs de risque, qui accumulés et combinés peuvent conduire l'individu vers la précarité. La précarité conserve par ailleurs sa double signification de risque, mais aussi facteur de risque, qui lorsqu'elle s'installe durablement, augmente la probabilité de devenir pauvre et/ou exclu.

Ainsi, ce sont les discontinuités de parcours subies, personnelles ou professionnelles, qui forment la précarité ou induisent son risque. C'est la représentation de cette précarité vécue ou potentielle, que nous souhaitons observer. Pour cela, nous focaliserons notre attention sur une population particulièrement exposée au danger de la précarisation, les étudiants. 


\section{La précarité, construction sociale d'un risque chez une population vulnérable}

Les étudiants ne se présentent pas comme un groupe homogène face au risque de précarité. La diversité des conditions de vie individuelles concourt en effet à une hétérogénéité d'exposition au danger, et cohabitent dans ce groupe des situations de précarité multiples et variées, conscientes ou inconscientes, latentes ou exprimées. Il nous faudra donc avant tout montrer que les étudiants peuvent être considérés comme un groupe social, car le risque est un construit culturel (Peretti-Watel, 2003), une production sociale (Le Breton, 2012), fruit des interactions individus-société, dans un temps et un espace donnés (Assailly, 1992). Cette formation collective et individuelle détermine un cadre de représentation du risque qui fonctionne comme un moyen d'assumer le danger (Orfali et Joffe, 2005).

\section{Les étudiants comme groupe social}

Le profil de l'étudiant qui n'a de préoccupation que ses études s'est profondément transformé. Si la condition étudiante était autrefois fortement marquée par la dépendance à l'égard de la famille, source première de logement et de moyens financiers (Erlich, 1998), l'étudiant d'aujourd'hui ne vit plus nécessairement au domicile familial, et lorsqu'il y vit, celui-ci ne joue plus nécessairement son rôle protecteur. Les configurations familiales se sont complexifiées, et les solidarités, bien que toujours présentes, se sont affaiblies.

De nombreux étudiants doivent donc trouver un emploi, à temps partiel pour la plupart, à temps plein pour certains, afin d'assumer leur vie étudiante, ou d'y participer lorsque la famille ne peut la prendre totalement en charge. Ses revenus doivent lui permettre de remplir son réfrigérateur, de payer son loyer et ses diverses factures (eau, gaz, électricité, téléphone voire internet), ses frais de déplacements, ses vêtements, et bien sûr, ses frais d'étudiant (inscription, livres, et matériels divers comme les stylos voire un ordinateur). Qu'ils dépendent de leur famille ou non, l'exercice d'une activité rémunérée concernera les trois-quarts des étudiants avant la fin de leurs études (Erlich, 1998). Si ce travail est pour certains vital, pour d'autres il répond à une volonté d'autonomisation voire à la préparation de l'insertion professionnelle. En effet, à mesure qu'ils avancent dans l'enseignement supérieur, les étudiants s'émancipent (autonomisation vis-à-vis de la famille) et se socialisent (leurs pratiques culturelles évoluent) : leurs besoins financiers augmentent. L'expérience de la vie active se pose alors comme une caractéristique nouvelle de la condition étudiante qui marque désormais son modèle culturel de référence (Erlich, 1998). Étudiant n'est pas un emploi, et pourtant l'étudiant qui cumule ses études avec une activité rémunérée se retrouve bel et bien victime d'une «double journée»: il exerce son métier d'étudiant (Coulon, 2000) tout en étant salarié par une autre organisation pour laquelle il travaille. Ce double rôle social augmente le risque d'échec aux examens, ce qui amène parfois à s'enferrer dans un cercle vicieux : ils sont étudiants pour trouver un 
«bon» travail, mais comme ils travaillent pour étudier, ils n'ont guère le temps d'accorder à leurs études toute l'attention qu'elles réclament. C'est donc, pour certains, une prise de risque qui se solde par un échec.

L'étudiant ne fait donc plus qu'étudier, il travaille pour payer ses dépenses et/ou réduire celles de sa famille. Par les responsabilités qui lui incombent et les difficultés quotidiennes auxquelles il doit désormais faire face, l'étudiant est entré de plein fouet dans les populations sensibles au risque de précarité. Il cumule en effet des difficultés liées aux trois méta-dimensions de la précarité : économique (contrats précaires, travail alimentaire, faible rémunération, difficulté à régler les dépenses), social (vie sociale et familiale) et psychologique (épuisement, stress, stigmatisation). Les étudiants présentent ainsi un temps et un espace propres et une gestion spécifique du quotidien (Erlich, 1998) qui permettent de les considérer comme un groupe social.

Ainsi, depuis la démocratisation de l'accès aux études et la multiplication des filières (Novi, 1997), la massification de ce groupe a engendré sa diversification interne et conduit à la mutation de ce groupe, mais sans pour autant le désagréger. En effet, avec une personne sur deux d'une même classe d'âge qui est étudiante et l'existence de caractéristiques communes en terme d'activités culturelles, économiques et de consommation, les étudiants tendent à former un groupe social (Coulon, 2000). Un groupe social est un ensemble d'individus possédant des caractéristiques sociales communes, relié par l'existence d'interactions, directes ou indirectes, entre leurs membres, et la conscience plus ou moins marquée d'une appartenance, de buts ou d'intérêts communs.

Le statut étudiant permet à la fois d'être reconnu comme un groupe social par les autres groupes, et d'asseoir au sein du groupe un sentiment d'appartenance. L'exercice d'une activité commune (les études), l'existence d'une finalité commune au groupe (l'obtention d'un diplôme), ou encore l'appartenance à une même organisation (l'enseignement supérieur), sont autant d'éléments permettant de justifier l'approche des étudiants comme un groupe social. Les étudiants forment ainsi un groupe social, non seulement au regard de critères endogènes, la présence d'une communauté d'intérêts et d'objectifs partagés par tous les étudiants, mais aussi exogènes puisque l'existence des étudiants en tant que groupe social va apparemment de soi dans le sens commun qui les identifie tantôt par leur statut, tantôt par la prolongation de l'état d'adolescence (Novi, 1997). La reconnaissance de ce groupe par le reste de la société entérine la considération de ce groupe comme un groupe social.

La question des frontières du groupe social se pose toutefois. En effet, peut-on considérer qu'un étudiant en fin de parcours scolaire tout comme un jeune faisant son entrée dans l'enseignement supérieur appartiennent pleinement à ce groupe ? 
Les étudiants commencent à s'extraire de ce groupe social quand ils arrivent en fin de parcours : l'étudiant s'autodéfinit alors moins par son statut de scolarisé que par son âge (Galland, 2011) ou encore son projet professionnel. Au même titre que les étudiants en fin de parcours, les jeunes qui font leur entrée dans l'enseignement supérieur sont dans une posture transitionnelle, du monde lycéen au monde étudiant. Ainsi pour ces jeunes étudiants, l'identité lycéenne subsiste car les liens tissés pendant leur période lycéenne perdurent en parallèle des nouvelles rencontres étudiantes et de l'apprentissage du métier d'étudiant (Romainville, 2001). L'appropriation de nouveaux codes, de nouvelles normes marque le passage entre ces deux mondes pour conduire à une affiliation au groupe social étudiant et à l'intégration de cette identité collective (Coulon, 2000).

Demême,peut-on considérerqu'unétudiantd'universitéappartientau même groupe qu'un étudiant d'école de commerce ? Soit : l'ensemble des scolarisés dans l'enseignement supérieur peut-il s'apparenter à un groupe social ? C'est une question qui fait encore débat. Bien que certaines différences aient déjà été relevées, notamment concernant la force de l'identité d'école comparée à celle d'UFR (notamment du fait d'intenses processus de socialisation amorcés dès l'arrivée du nouvel étudiant : l'intégration), d'autres recherches soulignent l'existence d'une conscience collective latente ou exprimée, notamment au regard des mobilisations étudiantes massives lorsque le groupe est menacé (Le Bart et Merle, 1997). Pour éviter un positionnement pour l'une ou l'autre de ces assertions, nous avons choisi de porter notre regard sur les seuls étudiants de l'enseignement supérieur universitaire. L'objectif est d'identifier et de comprendre les représentations individuelles et collectives du risque de précarité chez les étudiants. Il s'agira d'observer les représentations sociales du risque de précarité chez les étudiants mais aussi le risque subjectif de précarité chez les étudiants, soit leur évaluation personnelle du risque que représente la précarité.

\section{La représentation sociale du risque dans un groupe social}

Les représentations sociales sont des modalités de pensée pratique orientées vers la communication, la compréhension et la maîtrise de l'environnement social, matériel et idéel (Jodelet, 2003). Les représentations sociales portent sur un objet (concret ou abstrait : chose, personne, situation...) et permettent de construire et d'organiser la réalité sociale. Elles traduisent une manière de penser, d'interpréter et donc de se figurer le réel en donnant sens aux objets. Elles sont dites sociales car elles se construisent via les interactions individus-société, générant ainsi une forme de connaissance particulière, le savoir de sens commun, qui contribue à la formation d'une identité sociale source des regroupements et places des individus dans le champ social. Elles désignent ainsi une forme de connaissance socialement élaborée qui 
participe à la construction d'une réalité commune à un ensemble social, ou plus simplement, une forme de pensée sociale (Jodelet, 2003).

Une représentation sociale est constituée d'un double système, central et périphérique, qui organise sa structure et sa dynamique. La représentation sociale est composée d'un noyau central, autour duquel sont organisés des éléments dits périphériques (Abric, 2011 ; Moscovici, 1976). Le noyau central des représentations a deux fonctions principales : créatrice d'une part, car il est générateur de sens pour les autres éléments, et organisatrice d'autre part car il constitue le cœur de la structure représentationnelle permettant ainsi d'organiser et stabiliser la représentation. Le noyau central abrite tous les éléments qui donnent sens à la représentation : il décrit la nature de l'objet, le lien existant entre l'objet et le sujet (individu, groupe) et le contexte situationnel (normes sociales, système de valeurs, etc.). Ces éléments présentent alors deux dimensions : une dimension fonctionnelle avec les éléments centraux qui se rapportent directement à l'accomplissement d'une tâche, et une dimension normative avec les éléments centraux issus de normes, stéréotypes, habitudes, etc.

Le système périphérique joue un rôle primordial d'interface entre le noyau dur et l'environnement dans lequel la représentation se crée. Les éléments périphériques sont des «schèmes normaux», pointant ce qui est normal ou pas dans telle ou telle situation, qui peuvent se muer en «schèmes étranges» lorsqu'ils rentrent en conflit avec la réalité au travers par exemple de l'intégration de nouvelles pratiques contradictoires à la représentation. La modification de la représentation sociale ne peut ainsi se faire que par le biais de l'évolution des éléments périphériques, lesquels vont ensuite progressivement modifier la structure du noyau central pour alors transformer la représentation.

Dans la construction des représentations sociales, deux processus fondamentaux sont à l'œuvre : l'objectivation et l'ancrage (Moscovici, 1976). L'objectivation marque la constitution du noyau central de la représentation. C'est un processus par lequel l'individu va pouvoir s'approprier et intégrer des connaissances complexes : les informations sont triées en fonction des critères culturels et normes sociales actuelles, puis ces éléments sont agrégés en un noyau figuratif qui devient le centre autour duquel la représentation va se construire.

L'ancrage traduit l'appropriation de la représentation et de son objet par l'individu et la société. C'est à partir de ce processus que les représentations sociales vont pouvoir jouer leur rôle d'intégration de connaissances nouvelles, d'interprétation et construction du réel, et d'orientation des attitudes, pratiques et rapports sociaux.

La relative homogénéité des représentations du risque au sein d'un groupe relève de ce que Janis et Mann (1977) appellent la pensée de groupe. Elle est liée à des facteurs culturels, qui font référence à l'ensemble des normes, valeurs et croyances partagées par un groupe social (Tylor, 1876), ainsi qu'aux attributs fonctionnels et interprétatifs des représentations sociales, 
conduisant les individus à s'approprier l'identité de leur groupe. Cette tendance au mimétisme (Bandura, 1986) relève de la normativité du processus d'objectivation : les individus ajustent leurs représentations fonction de celles des autres (Cialdini, 1989) afin de se conformer au groupe et à son identité sociale. Les représentations des risques sont alors conçues comme le produit de processus culturels fondés sur l'objectivation et l'ancrage social. Les valeurs, croyances et normes sont des supports pour le groupe d'une classification des risques plus ou moins probables. Ainsi, « en choisissant un mode de vie, nous choisissons également de courir certains risques. Chaque forme de vie sociale a son propre portefeuille de risques. Partager les mêmes valeurs, c'est aussi partager les mêmes craintes, et inversement les mêmes certitudes » (Perretti-Wattel, 2003 citant Douglas et Wildawsky, 1982). L'étude des représentations sociales du risque de précarité chez les étudiants permet alors de révéler les attitudes qu'elles conditionnent à l'égard de la précarité et les comportements qu'elles orientent (Arciszewski et al., 2011).

Suite à la présentation du champ de la recherche, la formulation de propositions va permettre de cadrer notre étude empirique.

Nous retenons tout d'abord que la précarité est un processus multidimensionnel qu'il est complexe d'identifier. En revanche, il apparaît que ses facteurs de risque sont plus aisément saisissables. La précarité tout comme son évaluation devrait donc être représentée par des évocations liées à la santé, au logement, etc. Nous faisons donc la proposition suivante :

P1 : Les représentations sociales du risque de précarité chez les étudiants s'expriment avant tout selon ses facteurs de risque.

Nous retenons ensuite que les étudiants, entre dépendance et volonté d'autonomisation du cadre familial, sont considérés comme une population vulnérable au risque de précarité : leur probabilité de précarisation est forte. Lorsqu'un groupe social est menacé, s'opère une activation des liens de solidarité afin de protéger le groupe du reste de la société. Nous faisons donc la proposition que dans la gestion du risque de précarité, les étudiants considèrent que c'est la dimension sociale qui permet de venir en aide à un précaire.

P2: La gestion du risque de précarité chez les étudiants par des mesures de protection requiert une réactivation du lien social.

Ces propositions de recherche vont maintenant être étudiées au regard de l'analyse des données collectées sur le terrain.

\section{Méthodologie}

Un questionnaire publié en ligne accompagné d'une sollicitation par courrier électronique a été envoyé aux étudiants de l'Université de Pau et des Pays de l'Adour. La collecte des données, effectuée du 19 au 21 juin 2012 a permis de réunir 97 répondants ( 46 femmes, 44 hommes âgés de 19 à 25 ans, de L1 à M2). 
Nous avons privilégié l'élicitation libre afin de recueillir des données associatives (Di Giacomo, 1981) liées à l'objet de la représentation : la précarité. L'association libre " consiste, à partir d'un mot inducteur (ou d'une série de mots) à demander au sujet de produire tous les mots, expressions ou adjectifs qui lui viennent à l'esprit » (Abric, 2011). La spontanéité verbale permise par cette technique facilite le repérage du contenu de la représentation, et plus particulièrement l'émergence de son noyau central. Le questionnaire (cf. encadré 1), construit autour de nos deux propositions, était composé de quatre questions ouvertes contenant les mots inducteurs " précaire » et « précarité » et a permis le recueil de données principalement qualitatives.

1) Lorsque vous entendez le mot "précarité", qu'est ce que cela vous évoque?

2) Vous considérez-vous comme précaire ? Pourquoi ?

3) Existe-t-il dans votre entourage quelqu'un de précaire ? Si oui, pourriez-vous me parler de lui/elle?

4) Selon vous, que faut-il faire pour aider une personne précaire?

\section{Encadré 1: questionnaire}

La première question avait pour but de faire émerger le cœur des représentations sociales du risque de précarité, la deuxième et la troisième question de repérer les modalités d'évaluation de ce risque, et la quatrième d'observer les moyens de protection de ce risque. Le questionnaire était accompagné de questions socio-démographiques, visant à contrôler l'identité du répondant, mais aussi à procéder à une éventuelle segmentation du groupe.

Deux méthodes ont été mobilisées pour interpréter les données recueillies : l'analyse prototypique de Vergès (1992) afin de dresser une connaissance synthétique des représentations liées au risque de précarité, et l'analyse de contenu afin d'explorer plus finement le contenu et la structure de ces représentations. À cet égard, l'analyse de contenu est essentiellement centrée sur les occurrences et co-occurences des termes dans l'échantillon (logiciel Tropes ${ }^{6}$ ), ainsi qu'à leurs éventuelles correspondances avec les résultats obtenus dans la première question. L'analyse prototypique permet de faire apparaître la fréquence de chaque énonciation recueillie et de leur accorder un score calculé fonction de l'ordre d'apparition de ces évocations (score maximal de 5 pour le premier terme, score minimal de 1 pour le cinquième terme). L'analyse prototypique permet une approche synthétique de la représentation du risque de précarité, en révélant notamment le noyau central de la représentation caractérisé par les éléments les plus spontanément et fréquemment évoqués. Le prototype obtenu consiste en un tableau comprenant deux lignes, qui

6 Tropes est un logiciel développé par Pierre Molette et Agnès Landré sur la base des travaux de Rodolphe Ghiglione (http://www.tropes.fr/). 
séparent les verbatims à score élevés et faibles, et deux colonnes, qui séparent les verbatims à fréquence élevée et faible (la moyenne est utilisée comme seuil).

\section{Résultats et discussion}

\section{Le prototype du risque de précarité}

La collecte a permis de totaliser 390 évocations, pour une fréquence et un score moyens respectivement de 7,5 et 26 .

L'observation du noyau central de la représentation (tableau 1) met en évidence le profond ancrage de la précarité dans son origine sémantique : la pauvreté.

\begin{tabular}{|c|c|c|}
\hline Verbatim & Fréquence & Score \\
\hline Pauvreté & 59 & 262 \\
\hline Mal logé & 27 & 86 \\
\hline Chômage & 23 & 78 \\
\hline Problèmes financiers ponctuels & 20 & 72 \\
\hline Galère & 18 & 64 \\
\hline Mal-être & 13 & 35 \\
\hline Instabilité & 9 & 36 \\
\hline Jour le jour & 9 & 31 \\
\hline Rue & 8 & 32 \\
\hline
\end{tabular}

Tableau 1 : Noyau central de la représentation du risque de précarité

Évoqué 59 fois par les étudiants et quasi-systématiquement comme le premier mot qui leur vient à l'esprit lorsqu'ils entendent le terme " précarité », ce résultat peut être interprété de plusieurs façons. Il peut tout d'abord entériner la confusion qui existe entre les deux termes, bien souvent (et à tort) considérés comme des synonymes. Toutefois, hormis l'évocation de la « rue », qui traduit là une confusion avec la notion d'exclusion, il semblerait que la précarité ne soit ensuite pas amalgamée à ces deux notions voisines. La force du terme "pauvreté » lorsque la précarité est évoquée pourrait alors provenir de sa conception bien identifiée comme risque de pauvreté lorsqu'elle tend à devenir durable.

Le noyau central révèle ensuite que la précarité est appréhendée dans ses diverses formes : les étudiants se représentent la précarité via ses facteurs de risque. Deux facteurs de risque sont ainsi profondément ancrés dans l'esprit des étudiants : la mauvaise qualité du logement et l'absence d'emploi. La précarité est ensuite décrite de manière plus générale, et c'est alors la conception de la précarité en tant que risque qui apparaît nettement : les problèmes financiers ponctuels, l'instabilité, la galère, et la gestion de sa vie au jour le jour, avec 
le risque de voir sa situation se dégrader. La précarité représente pour les étudiants le mal-être, la souffrance, la détresse.

L'observation du verbatim " chômage " dans le noyau central de la représentation interpelle. En effet, ce facteur de risque lié au monde du travail suggère une inquiétude des étudiants quant à leur avenir professionnel. Sa forte présence dans l'esprit des étudiants pourrait par ailleurs expliquer que nombre d'entre eux ne se considèrent pas comme précaires (cf. analyse de contenu). En effet, les étudiants n'étant pas encore pleinement exposés à ce facteur de risque, ils peuvent sous-évaluer leur risque de précarité du fait de leur association de la précarité au chômage.

La proposition 1 apparaît donc comme incomplète. Si les étudiants se représentent bien le risque de précarité via ses facteurs de risque, ils semblent avant tout considérer la précarité comme un facteur de risque de pauvreté.

Par ailleurs, comme enseigné dans la littérature, l'aversion au risque de précarité est globalement forte. La précarité n'est aucune fois saisie comme positive : la mobilité, la flexibilité, les opportunités engendrées par le changement, etc. ne sont purement et simplement pas évoquées. C'est bien la «précarité à la française» qui s'exprime ici. Toutefois, une prémisse de tolérance au risque de précarité semble émerger. L'évocation du «système», de «la crise» ou encore du caractère «courant» de la précarité, suggère le sentiment d'impuissance des étudiants. Ces verbatims restent marginaux et ne sont donc pas ancrés dans le noyau central, mais leur position dans le système périphérique des représentations sociales soulève malgré tout une question préoccupante : la représentation sociale du risque de précarité chez les étudiants va-t-elle, ou est-elle déjà en train de se reconstruire autour de cet élément?

\section{L'analyse de contenu des représentations du risque de précarité}

\section{L'évaluation du risque de précarité}

À la question préliminaire "vous considérez-vous comme précaire ? ", 10 étudiants seulement sur 70 répondent par l'affirmative. Pour eux, la précarité est avant tout un combat quotidien pour continuer de mener leurs études (cf. encadré 2).

Ainsi 7 d'entre eux soulignent la difficulté de leur situation en usant d'expression marquant la récurrence de leurs problèmes («tous les jours», «chaque mois», etc.) ou l'insuffisance des aides lorsqu'ils en reçoivent. Les références à l'argent apparaissent 15 fois, et les verbes «devoir» et «ne pas pouvoir» apparaissent 8 fois, et témoignent bien de la souffrance de ces étudiants. Ils doivent se battre quotidiennement pour mener à bien leurs études tout en essayant de maintenir un niveau de vie décent. Pour cela ils gèrent avec précision et leur temps et leur argent. Ces étudiants ne bénéficient pas ou très peu de la solidarité familiale. Pour vivre ils sont alors dépendants 


\begin{abstract}
« je suis étudiante, je dois faire face à des responsabilités vis-à-vis de mes études qui peuvent être endommagées suite à des difficultés financières. Je suis contrainte de travailler durant l'année pour pouvoir vivre et cela a des incidences sur mes études les conditions de travail n'étant pas optimales pour étudier. De plus l'université nous oblige à faire un certain nombre de dépenses importantes mais auxquelles nous ne pouvons pas pallier (ex l'achat d'un ordinateur...) que tout le monde ne peut pas se permettre.» [Étudiante, 23 ans, M2, UFR Sciences et technologies, Pau] «je n'ai pas de revenus réguliers, que je me pose chaque mois la question «comment je vais payer mon loyer, mes dépenses», que je dois me restreindre sur mes dépenses de nourriture, que je ne peux faire aucun projet à cause de cette situation, par ailleurs génératrice d'angoisses. Parce que j'ai l'impression que peu de personnes autour de moi comprennent la réalité de la situation, et que je doute parfois d'avoir un jour une situation meilleure » [Étudiante, 27 ans, M2, UFR Arts, lettres, langues, sciences humaines et sociales, $\mathrm{Pau}$ ]
\end{abstract}

\title{
Encadré 2: verbatims d'étudiants précaires
}

de l'aide sociale, ou contraints de trouver un emploi rémunéré. Notons par ailleurs que parmi ces 10 étudiants, la moitié est effectivement en retard dans ses études au regard de son âge, avec au minimum deux ans de retard. Sauf choix volontaire (réorientation), cela tendrait à confirmer la difficulté de ces étudiants précaires à concilier leur emploi rémunéré avec une conduite sereine de leurs études.

L'analyse globale des réponses permet de mettre en évidence la forte occurrence du connecteur additionnel «et» lors de l'autoévaluation de la précarité (53 occurrences). La contextualisation permet de repérer que ces connecteurs additionnels sont positionnés entre les divers facteurs de risque que l'étudiant mobilise pour justifier de sa situation Contrairement aux résultats prototypiques, ces éléments correspondent à une représentation du risque de précarité par l'analyse du cumul de ses facteurs de risque. Par ailleurs, les modélisations d'intensité et de négation sont fortement présentes et liées aux mêmes verbatims. La modulation de l'intensité (52 occurrences) s'opère principalement via une atténuation du discours («quelques», "pas beaucoup», «pas très», etc.). La présence d'éléments de négation («n'ai pas», «ne me sens pas», «ni», etc.) est quant à elle fortement corrélée aux facteurs de risque de précarité, soit qu'ils soient eux même l'objet de la négation, soit qu'ils soient évoqués mais que la précarité soit tout de même niée (46 occurrences). Cela tend à mettre en évidence une certaine forme de minimisation du risque de précarité ou de l'influence de ses facteurs. Le faible nombre de précaires déclarés pourrait alors correspondre à une sous-évaluation du risque de 
précarité. En effet, au regard des réponses de certains non-précaires déclarés, leur situation s'apparente pourtant à une précarisation avérée (cf. encadré 3).

«Malgré certaines privations, je ne me sens pas malheureuse dans ma situation. Je ne suis pas dans le besoin même si je rencontre certaines fois des difficultés financières. Il faut savoir se satisfaire de ce qu'on a avant de vouloir ce qu'on n'a pas » [Étudiante, 23 ans, M1, UFR DEG, Pau]

Encadré 3: verbatim d'une étudiante objectivement précaire

Biais d'optimisme (Peretti-Watel, 2001) ? Refus de la stigmatisation ? En tout cas cela révèle que la précarité est avant tout un risque subjectif, et que la seule appréciation de la combinaison des facteurs de risque ne suffit pas à repérer la précarisation. L'objectivation de ce risque, par le calcul d'un score rattaché à des événements purement factuels, tel que le score Epices par exemple, est insuffisante pour apprécier le risque de précarité.

La présence de certaines sécurités permet à de nombreux étudiants de ne pas se considérer comme précaires ou de ne pas craindre la précarisation : les aides sociales, mais aussi et surtout les parents ou encore l'exercice d'une activité rémunérée. Les parents comptent comme la plus fréquemment évoquée. Toutefois, si elle a pu être naturelle, la solidarité familiale est désormais considérée de manière flagrante comme une " chance ». Certains étudiants sont aussi conscients de la fragilité qu'induit cette dépendance (cf. encadré 4).

« J'ai des parents qui m'aident financièrement. Autrement les cours particuliers ou juste le travail saisonnier et actuellement la paie de stage ne suffiraient pas à satisfaire mes besoins élémentaires (logement, nourriture, assurance,...) » [Étudiant, 23 ans, M2, Autre, Pau]

Encadré 4 : verbatim d'une étudiante dépendante à l'aide parentale

Ces étudiants dépendant de leurs parents, parce qu'ils vivent au domicile parental ou parce que ceux-ci les aident matériellement, estiment souvent cette chance au regard de la non obligation de travailler en parallèle de leurs études. L'exercice d'une activité rémunérée en parallèle des études est alors appréhendé comme un facteur de risque de précarité, mais il peut aussi être vécu comme une sécurité par certains étudiants. Ceux qui travaillent ne se considèrent généralement pas comme précaires car ce sont ces revenus qui leurs permettent d'assurer leurs conditions de vie. Pour les étudiants dépendants des parents, le travail représente une difficulté augmentant leur probabilité de rentrer dans la précarité, notamment parce qu'ils ne se sentiraient pas capables de concilier leurs études avec l'exercice d'une activité rémunérée 
(cf. encadré 5). Le travail étudiant est ainsi à la fois considéré comme un facteur de risque de précarité et un moyen de prévention de ce même risque.

« Mes parents ont un travail stable (ouvrier et fonctionnaire) et sans toucher un salaire de ministre ils arrivent à subvenir à mes besoins (payer mes études, mon appartement) sans que j’aie besoin de travailler, ce qui me permet de rester concentrée " [Étudiante, 20 ans, L2, UFR DEG, Pau]

Encadré 5: verbatim d'une étudiante concentrée sur ses seules études

Le statut d'étudiant est lui aussi controversé. Si pour certains, être étudiant signifie être exposé aux dangers de la précarité, pour d'autres ce statut est au contraire une réponse en elle-même à la demande d'autoévaluation de leur précarité. Ainsi à l'amalgame entre précarité et statut d'étudiant (" même si je suis étudiant, pour telle et telle raisons je ne suis pas précaire ») s'oppose une vision complètement inverse, le statut étudiant devenant la cause du non risque de précarité ( je suis étudiant, donc je ne suis pas précaire»).

Cette analyse de contenu nous révèle que les termes les plus fréquemment évoqués sont liés à l'argent («argent», "payer», «revenus», etc.) avec 49 occurrences. Il est un repère permettant aux étudiants d'autoévaluer leur précarité. Soit qu'il soit lié aux revenus du travail, soit aux aides sociales, soit à l'aide parentale, l'argent permet aux étudiants de gérer le quotidien et donc d'appréhender leur risque de précarité par leur capacité à subvenir à leurs besoins. Là encore, il faut s'interroger sur l'ancrage de la précarité dans son origine sémantique, la pauvreté. Pour autant, l'argent est moins évoqué pour son manque que pour son insuffisance ponctuelle, sa difficulté à gérer son budget, ce qui caractérise effectivement l'instabilité définissant la précarité. La confusion observée dans le noyau central du prototype n'est donc pas entérinée par l'analyse de contenu. Ensuite, ce sont l'univers de la famille (37 occurrences) et de l'éducation (43 occurrences) qui apparaissent le plus souvent dans le discours. La famille est évoquée comme source de dépendance de l'étudiant : elle lui procure un logement, aide financière et lien social. La co-occurrence entre mots la plus fréquente est celle de l'association «parents-chance»(7 co-occurrences) démontrant que dans l'esprit des étudiants, la solidarité familiale n'est plus la norme, la famille ne joue plus de manière naturelle son rôle protecteur. Le thème de l'éducation s'exprime quant à lui massivement via le statut étudiant, considéré soit comme une protection, lorsque l'étudiant dépend de sa famille, soit comme un risque de précarité lorsqu'il est indépendant, et c'est alors la conciliation difficile des études et d'un travail rémunéré qui est évoquée. La prégnance de ces deux 
thèmes montre bien la situation de tension dans laquelle se trouve l'étudiant, entre dépendance et volonté d'autonomisation.

Les dimensions du risque de précarité apparaissent enfin via le travail et l'emploi (23 occurrences), le logement (17 occurrences) et l'alimentation (14 occurrences). Ces trois thèmes sont ainsi bien moins présents dans l'autoévaluation du risque de précarité que dans son prototype. Notons enfin que le champ lexical de la dépendance («prise en charge», «aider», etc.) et des problèmes («difficultés», «situation difficile», etc.) sont relativement présents (avec respectivement 23 et 15 occurrences).

Enfin, pour approfondir l'autoévaluation de la précarité, la représentation de ce risque a aussi été approchée par une évaluation de «l'autre », du précaire que l'on côtoie, afin de vérifier la concordance des éléments représentationnels du risque de précarité. Cette question pourrait s'assimiler à une technique projective, telle qu'elle est pratiquée en psychologie.

Peu de répondants déclarent connaître une personne précaire : seulement 19 étudiants. Cette faiblesse des réponses pourrait signifier soit que les étudiants connaissent effectivement peu de personnes dans cette situation, soit qu'il reste très difficile pour l'étudiant d'évaluer ce risque lorsqu'il ne le touche pas personnellement. Il est en effet difficile de repérer la précarité des autres quand celle-ci comporte une part de subjectivité. Pour cela, les précaires identifiés par les étudiants le sont majoritairement au regard de critères objectifs, les facteurs de risque liés à l'emploi ou à la dépendance aux aides sociales étant majoritairement évoqués. Sur les 19 personnes précaires évoquées, 7 appartiennent à la famille proche de l'étudiant. Pour ces étudiants, le risque de précarité augmente car la capacité protectrice de la famille est moindre et ne peut être identifiée comme une sécurité pour l'étudiant. Par ailleurs, soulignons que sur les 10 étudiants précaires, la moitié connaît une personne précaire. Cela pourrait signifier que les étudiants précaires sont plus enclins à repérer les symptômes de la précarité, mais c'est un sujet qui mériterait plus d'investigation. Dans notre échantillon, deux étudiants apparaissent en grande précarité : non seulement ils se considèrent précaires, mais ils identifient en plus comme tels leur proche famille (le frère pour l'un, les parents pour l'autre).

\section{Mesures de protection du risque de précarité}

À la question "Selon vous, que faut-il faire pour aider une personne précaire ?", 64 étudiants se sont exprimés. Les réponses sont massivement orientées vers des moyens de protection liés aux méta-dimensions psychologiques et sociales du risque de précarité.

La protection du risque de précarité est avant tout une question de lien social. Cette thématique est évoquée plus de 8 fois sur 10 par les répondants. La recomposition du lien social passe selon les étudiants par un soutien, une écoute, une aide. C'est une question de solidarité (10 occurrences). Les 
termes «aider» et «aide(s)» sont les plus prégnants (43 occurrences) et sont majoritairement employés dans le sens d'accompagner. Cet accompagnement se décline dans les différents gestes du quotidien (cf. encadré 6) et touche au vocabulaire de l'éducation, de l'accès à l'information, de l'orientation, afin de prévenir le risque d'une installation durable de cette précarité car celle-ci « laisse s’installer la tristesse, la démotivation, le laisser-aller, choses néfastes pour sortir de cette situation».

« Être présent, essayer de la faire tenir moralement, avoir un suivi sur cette personne, l'aider à trouver un emploi, la guider vers tous les organismes qui peuvent l'aider, lui donner de quoi vivre (vêtements, nourriture etc.) » [Étudiante, 23 ans, M1, UFR DEG, Pau]

Encadré 6: verbatim d'une étudiante proposant un accompagnement multidimensionnel

Cela met en exergue toute la difficulté de s'attaquer à la résorption de la précarité. Multiforme, d'intensité variable, inverser le processus de précarité n'est pas chose aisée, mais il est clair pour les étudiants qu'il est « essentiel que la personne ne se sente pas seule » pour ne pas qu'elle tombe dans l'exclusion. S'occuper de la précarité c'est aussi prévenir le risque d'exclusion, à condition de " l'aider à s'en sortir sur le long terme et non sur du court terme ». Il est important d'insister sur le fait que cette aide n'est pas conçue comme unilatérale, elle est le fruit d'une collaboration avec le précaire qui accepte d'être épaulé dans la gestion de ses difficultés. L'aide n'est pas un droit, elle doit émaner de la rencontre de deux volontés, celle de l'aidé et celle de l'aidant. Il transparaît alors dans certaines réponses, pourtant charitables, une forte stigmatisation latente qui ne s'observait pas dans les réponses précédentes. Il faut aider la personne précaire mais, il faut « avant tout savoir si leur condition leur convient ou non » ou encore " que cette personne souhaite s'en sortir». Ainsi, bien que certains étudiants soient précaires au regard de l'évaluation objective du risque de précarité, ils ne se considèrent pas comme tels. Du coup, lorsqu'il s'agit de «l'autre», il semblerait que certains étudiants soient plus sévères (cf. encadré 7).

Certes ces étudiants proposent du soutien, de l'écoute, de l'accompagnement, mais dans leurs réponses transparaît une figure du précaire qui gère mal son budget, qui mange mal, qui boit, ou encore qui fraude pour obtenir des aides sociales. Dans le même esprit, d'autres vont plus loin et font l'apologie de la responsabilisation : il est inutile d'apporter son aide, la personne précaire est responsable de sa situation, c'est à elle de l'assumer. La responsabilité est une notion corrélée à celle de risque (Le Breton, 2012), et c'est bien ce que confirment certains étudiants en signifiant que si une personne est précaire, c'est qu'elle a mal géré le risque. Ces étudiants restent minoritaires dans notre 
échantillon (5 étudiants sur 64) mais contrastent fortement avec la vision solidaire qu'ont exprimée leurs pairs.

«Très compliqué ! Revoir les priorités de loisirs, moins consommer d'alcool, cuisiner soi-même, covoiturage voire faire stop,... Lui apporter des conseils : formation (notamment rédaction $\mathrm{CV}$, lettre de motivation et pack office) intellectuelle et manuelle » [Étudiant, 23 ans, M2, Autre, Pau]

«La solution n'est pas l'assistance mais plutôt l'assurance selon moi. Il faut laisser l'individu se prendre en main, qu'il arrête de penser que c'est à l'État de subvenir à ses besoins. » [Étudiante, 19 ans, L3, UFR DEG, Pau]

Encadré 7: verbatim d'étudiants portant un jugement

Par ailleurs, si les méta-dimensions psychologiques et sociales du risque de précarité constituent les principaux moyens de protection pour les étudiants, la dimension économique via l'emploi reste aussi un moyen d'aide non négligeable. L'emploi est ainsi évoqué 33 fois, comme source de cette précarité et moyen de la réduire, et les moyens financiers 17 fois, mais toujours avec un accompagnement ("l'aider à trouver un emploi », "l'aider à se reconstruire d'un point de vue social et financier », etc.).

\section{Conclusion}

Les limites de cette recherche tiennent à la visée exploratoire de la démarche. Pour dévoiler les représentations sociales du risque de précarité chez les étudiants, une méthodologie qualitative a été adoptée et a permis de recueillir les associations libres de 97 étudiants. Si la taille de l'échantillon n'est pas source de limite, sa composition en revanche impose une limite de validité externe des résultats présentés (représentativité). Ceux-ci appellent donc à être confirmés par des travaux complémentaires, ce qui nous permet de souligner les principaux enseignements de cette étude puis d'introduire les perspectives de prolongement de cette recherche.

Il est tout d'abord important de rappeler que sur cet échantillon (97 étudiants), la grande majorité ne se considère pas précaire. L'analyse de contenu de leurs évocations révèle pourtant que leur accumulation de nombreux facteurs de risque de précarité les rend vulnérables. La négation du risque pourrait résulter d'une sous-évaluation du risque de précarité, notamment liée à la crainte de la stigmatisation. Enfin, 10 étudiants se reconnaissent précaires. Il ne nous appartient pas de juger de la force ou de la faiblesse de cette quantité, la présentation des conditions de vie de certains de ces étudiants (cf. encadré 2) se suffisant à elle-même.

La discussion de la première proposition ("Les représentations sociales du risque de précarité chez les étudiants s'expriment avant tout selon ses facteurs 
de risque ») ne nous permet pas de prendre une position affirmée quant à la confirmation ou l'infirmation de celle-ci. Le cœur de la représentation sociale ne met en évidence que deux facteurs de risque de précarité, la mauvaise qualité du logement et le chômage, et le terme " précarité » est avant tout associé à " pauvreté ». L'analyse de contenu en revanche, dévoile de nombreux facteurs de risque de précarité supplémentaires. Les représentations sociales du risque de précarité chez les étudiants sont ainsi autant liées à ses facteurs de risque, qu'à la précarité en tant que facteur de risque de pauvreté et d'exclusion. La proximité sémantique entre ces trois termes et leur utilisation galvaudée conduisent à la construction et à la représentation d'un risque de précarité aux frontières extensives.

La discussion de la seconde proposition (« la gestion du risque de précarité chez les étudiants par des mesures de protection requiert une réactivation du lien social ») se révèle plus claire. Il apparaît en effet nettement que dans l'esprit des étudiants, la gestion du risque de précarité par des mesures de protection demande une réactivation du lien social. L'accompagnement, le soutien, l'écoute doivent avoir pour but de rendre son autonomie et sa dignité à la personne précaire. L'atteinte de ces objectifs nécessite un suivi régulier, transversal et de longue haleine. Cela nous permet d'avancer que pour protéger mais aussi prévenir les étudiants du risque de précarité, il faut avant tout s'attacher à étoffer le lien social, pour favoriser le développement de solidarités informelles. La proximité relationnelle au sein du groupe social permettrait, outre le développement de l'entraide et le soutien de l'étudiant par ses pairs, de repérer, en amont de la précarisation, les étudiants sensibles à ce risque. La socialisation serait ainsi un moyen de gestion du risque de précarité chez les étudiants, à la fois au niveau de sa prévention et de sa protection.

Afin de prolonger cette recherche, une prochaine étude pourrait notamment affiner l'observation des représentations sociales du risque de précarité chez les étudiants en procédant à une segmentation de ce groupe social. La question de la pertinence du groupe social étudiant est en effet soulevée par la littérature, et l'exploration des données interroge quant à l'éventuelle opportunité de relever les différences représentationnelles du risque de précarité selon certains critères socio-démographiques. Procéder à des regroupements intra-groupe pourrait notamment se révéler intéressant concernant le niveau d'étude, et plus particulièrement les étudiants situés aux deux extrémités du statut (L1 et M2). Ce passage du lycéen à l'étudiant ou de l'étudiant au futur travailleur entraîne une perte de repères qui conduit à l'ambiguïté de son identité. L'enjeu est alors de comprendre comment et en quoi la présence de ces trois types d'étudiants (les étudiants, les étudiants-lycéens et les étudiants-catégorie professionnelle en devenir), au sein d'un même groupe social, confronte des représentations et des attitudes différentes à l'égard du risque de précarité. Outre le niveau d'étude, deux critères de segmentation supplémentaires auraient été intéressants : l'exercice d'une activité rémunérée durant la période 
universitaire et le degré d'indépendance à l'aide familial (logement, aide financière...). L'intégration de ces données dans une prochaine recherche est apparue fondamentale et devrait permettre d'affiner la compréhension de la représentation du risque de précarité chez les étudiants pour mettre à jour les mesures de prévention et protection les mieux adaptées à la gestion du risque de précarité chez les étudiants.

\section{BIBLIOGRAPHIE}

ABRIC J.-C., Pratiques sociales et représentations, Paris, Presses Universitaires de France, 2011.

ARCISZEWSKI T., HARMA K., GOMBERT A., ROUSSEY J.-Y., « Effet de la visibilité du handicap et de l'expérience d'intégration sur la représentation sociale du handicap chez de jeunes collégiens ", Travail et formation en éducation, 8, 2011.

ASSAILLY J.-P., Les jeunes et le risque : une approche psychologique de l'accident, Paris, Vigot, 1992.

AUBERT N., L'individu hypermoderne, Paris, Erès, 2004.

BANDURA A., Social foundations of thought and action: a social cognitive theory, New-York, Prentice-Hall, Englewood Cliffs, 1986.

BARBIER J.-C., «La précarité, une catégorie française à l'épreuve de la comparaison internationale ", Revue française de sociologie, 2, 46, 2005, p. 351-371.

BAVEREZ N., «La spécificité française du chômage structurel de masse, des années 1930 aux années 1990 », Vingtième Siècle. Revue d'histoire, 1, 52, 1996, p. 41-65.

BECK U., " Le conflit des deux modernités et la question de la disparition des solidarités ", Lien social et politiques, 39, 1998.

BECK U., La société du risque: Sur la voie d'une autre modernité, Paris, Flammarion, 2008.

BOUMAZA M., PIERRU E., « Des mouvements de précaires à l'unification d'une cause ", Sociétés contemporaines, 1, 2007, p. 7-25.

CASTEL R., Les Métamorphoses de la question sociale, Paris, Gallimard-Jeunesse, 1999.

CASTEL R., L'Insécurité sociale : Qu'est-ce qu'être protégé ??, Paris, Seuil, 2003.

CIALDINI R.-B., Social motivations to comply: norms, values, and principles, Philadelphie, University of Pennsylvania Press, 1989.

CLERC D., La France des travailleurs pauvres, Paris, Hachette Littératures, 2009.

COULON A., Le Métier d'étudiant: l'entrée dans la vie universitaire, Paris, Presses Universitaires de France, 2000.

DI GIACOMO J.-P. (1981), " Aspects méthodologiques de l'analyse des représentations sociales ", Cabiers de Psychologie Cognitive, 4, 1, 1981, p. 397-422.

EHRENBERG A., L'Individu incertain, Paris, Hachette Littérature, 1999.

EHRENBERG A., La Fatigue d'être soi. Dépression et société, Paris, Odile Jacob, 2000.

EHRENBERG A., Le culte de la performance, Paris, Hachette Littératures, 2008.

ERLICH V., Les nouveaux étudiants, un groupe social en mutation, Paris, Armand Colin, 1998. 
GALLAND O., Sociologie de la jeunesse, Paris, Armand Colin, 2011.

GIDDENS A., Les conséquences de la modernité, Paris, L'Harmattan, 2000.

HÉLARDOT V., «Précarisation du travail et de l'emploi : quelles résonances dans la construction des expériences sociales? », Empan, 4, 60, 2005, p. 30-37.

JODELET D., Les représentations sociales, Paris, Presses Universitaires de France, 2003.

LE BART C. L., MERLE P., La citoyenneté étudiante: Intégration, participation, mobilisation, Paris, Presses Universitaires de France, 1997.

LE BRETON D., Sociologie du risque, Paris, Presses Universitaires de France, 2012.

MALINVAUD E., "Les causes de la montée du chômage en France », Revue française d'économie, 1, 1, 1986, p. 50-83.

MOSCOVICI S., La Psychanalyse, son image et son public, Paris, Presses Universitaires de France, 1976.

MOULIN J.-J., LABBE É., SASS C., CHATAIN C., GERBAUD L., « Précarité et facteurs de risque : le score Epices ", Revue Francophone de Psycho-Oncologie, 2, 5, 2006, p. $115-121$.

NOVI M., «Les difficultés de la vie étudiante : effets d'intégration et effets d'origine ", Cabiers de l'Urmis, 2-3, 1997, p. 17-28.

ORFALI B., JOFFE H., « De la perception à la représentation du risque : le rôle des médias ", Hermès, 41, 2005, p. 121-129.

PERETTI-WATEL P., La société du risque, Paris, La Découverte, 2001.

PERETTI-WATEL P., Sociologie du risque, Paris, Armand Colin, 2003.

ROMAINVILLE M., L'échec dans l'université de masse, Paris, L'Harmattan, 2001.

SANCHOU C., Introduction, Empan, 4, 60, 2005, p. 10-13.

SASS C., GUÉGUEN R., MOULIN J.-J., ABRIC L., DAUPHINOT V., DUPRÉ C., GIORDANELLA J.-P., « Comparaison du score individuel de précarité des Centres d'examens de santé, EPICES, à la définition socio-administrative de la précarité ", Santé Publique, 4, 18, 2006, p. 513-522.

JANIS I. L., MANN L., Decision making, a psychological analyses of conflict, choice and commitment, New York, The Free Press, 1977.

SPECHT M., "La pensée résiliente », Les cahiers internationaux de psychologie sociale, 78, 2, 2008, p. 79-94.

TYLOR E. B., La civilisation primitive, Paris, C. Reinwald, 1876.

VERGÈS P., « Lévocation de l'argent : une méthode pour la définition du noyau central d'une représentation ", Bulletin de psychologie, 405, 45, 1992, p. 203-209.

WRESINSKI J., Grande pauvreté et précarité économique et sociale, Journal officiel du 28 février, Paris, Conseil économique et social, 1987.

Résumé : La précarité s'est imposée comme un risque social majeur dans la société française. Les étudiants, entre dépendance et volonté d'autonomie, sont particulièrement exposés aux dangers de la précarité. Comprendre la représentation de ce risque pourrait alors permettre d'agir en amont de la précarisation, pour protéger mais aussi prévenir son amorçage chez les étudiants. Une analyse prototypique et une analyse de contenu mettent en évidence que les étudiants identifient la précarité par ses multiples facteurs de risque 
mais aussi comme un risque de pauvreté et d'exclusion. Si le mal-logement, le chômage ou les difficultés financières ponctuelles apparaissent comme les dimensions principales de ce risque, la réactivation du lien social serait le meilleur moyen de prévention et protection pour diminuer le risque de précarité chez les étudiants.

Mots-clés : risque de précarité, risque de pauvreté et d'exclusion, représentations sociales, protection du risque, prévention du risque, évaluation du risque.

\begin{abstract}
Precarity has become a major social risk in French society. Students, between dependence and will of autonomy are particularly exposed to the dangers of precarity. Understanding the representation of this risk could then allow to act upstream of the impoverishment process, to protect but also to prevent its apparition among students. A prototypical analysis and a content analysis highlight that students identify precarity through its multiple risk factors but also as a risk of longstanding poverty and exclusion. If unsane housing, unemployment or unexpected financial diffculties appear as the main dimensions of this risk, the reactivation of social connections might be the best means of prevention and protection against the risk of precarity among students.
\end{abstract}

Keywords : Risk of precarity, Risk of poverty and exclusion, Social representations, Risk protection, Risk prevention, Risk assessment. 